\title{
O-GlcNAcylation Enhances NUSAPI Stability and Promotes Bladder Cancer Aggressiveness
}

This article was published in the following Dove Press journal: OncoTargets and Therapy

\section{Yifan Chen* \\ Ji Liu (1)* \\ Wentao Zhang (D) \\ Aimaitiaji Kadier* \\ Ruiliang Wang (DD \\ Haimin Zhang \\ Xudong Yao (iD)}

Department of Urology, Shanghai Tenth People's Hospital, Tongji University of Medicine, Shanghai, 200072, People's Republic of China

*These authors contributed equally to this work
Correspondence: Xudong Yao; Haimin Zhang

Department of Urology, Shanghai Tenth

People's Hospital, Tongji University of

Medicine, Shanghai 200072, People's

Republic of China

Email yaoxudong1967@I63.com;

zhm1064@163.com
Objective: NUSAP1 and O-GlcNAcylation were reported to be hyper-activated in many kinds of cancers and involved in the advanced progression of cancers. In bladder cancer, O-GlcNAc transferase (OGT) expresses in patients' urine samples, with no expression in healthy individuals, indicating O-GlcNAcylation might involve in the occurrence and development of bladder cancer. Therefore, the present study aims to investigate the effects of O-GlcNAcylation in bladder cancer and if it can regulate NUSAP1 protein.

Materials and Methods: Western blot, immunohistochemistry, and PCR were used to evaluate the protein expression and mRNA level of NUSAP1; CCK-8 and flow cytometry used to evaluate the proliferation and inhibited the apoptosis of bladder cancer.

Results: The results showed that NUSAP1 was highly expressed in bladder cancer cells and tissue samples. NUSAP1 up-regulation significantly promoted the proliferation and inhibited the apoptosis of bladder cancer HT-1376 and T24 cells. Besides, the expression of O-GlcNAc was elevated in bladder cancer tissues and cells, and up-regulation of O-GlcNAc with GlcNAc and PuGNAc obviously increased NUSAP1 protein expression and stability. Moreover, knockdown OGT significantly inhibited the proliferation and tumorigenesis and promoted the apoptosis of bladder cancer cells, confirmed by CCK-8, in vivo xenotransplantation, and flow cytometry, whereas these roles were impaired when NUSAP1 was up-regulated.

Conclusion: Overall, our study makes clear that hyper-O-GlcNAcylation accelerates bladder cancer progression through promotion of NUSAP1 expression and its stability.

Keywords: NUSAP1, O-GlcNAcylation, stability, bladder cancer, aggressiveness

\section{Introduction}

Bladder cancer is the most common malignant tumor among urogenital system carcinomas, with gradually increasing incidence in recent years influenced by factors such as smoking and environmental pollution worldwide. ${ }^{1}$ In global male cancers, the incidence and mortality of bladder cancer rank sixth and ninth, respectively. $^{2}$ Radical cystoprostatectomy bilateral pelvic lymphadenectomy and urinary diversion are the gold standard for the treatment of male MIBC. However, patients often have distant recurrence after surgery, and the treatment effect is not satisfactory. Therefore, it is an important and even preferred way to fully understand and master the development mechanism of bladder cancer to find new effective therapeutic targets.

NUSAP1, first discovered in 2003, is a microtubule-binding protein mainly located in the nucleolus, with a molecular weight of about $55 \mathrm{KD}$. In recent years, numerous studies have found that NUSAPl is highly expressed in a variety of kinds of tumors and participates in their development, including astrocytoma, ${ }^{3}$ renal cell carcinoma, ${ }^{4}$ 
oral squamous cell carcinoma, ${ }^{5}$ prostate cancer, ${ }^{6,7}$ breast cancer, $^{8}$ and so on. For instance, Gulzar et $\mathrm{al}^{9}$ found that the recurrence of tumor after prostatectomy was closely associated with the increased transcription level of NUSAPl. Besides, Gordon et $\mathrm{al}^{6}$ suggested that upregulation of NUSAP1 significantly enhanced cell invasion and migration. Similarly, NUSAP1 was found to be overexpressed in $68.78 \%(152 / 221)$ of astrocytoma tissues, and the high level of it showed significant association with patients' poor survival, which also obviously promoted the invasion of astrocytoma cells. ${ }^{3}$ These findings all indicated that NUSAP1 might function as an oncogene in cancer. However, the expression pattern and effects of NUSAP1 in bladder cancer still remains unknown.

Glycosylation is a major post-transcriptional protein modification, which plays an important role in many biological processes. More than $50 \%$ of human proteins have been identified can be glycosylated, ${ }^{10}$ followed by enhanced stability. ${ }^{11,12}$ O-Linked $\beta$-N-acetylglucosamine (O-GlcNAc) is a main kind of glycosylation for cytosolic and nuclear proteins through covalent addition of a GlcNAc sugar moiety to the serine and/or threonine residues of proteins, ${ }^{13}$ which is regulated by O-GlcNAc transferase (OGT) and O-GlcNAcase (OGA) ${ }^{14-16}$ OGT transfers the GlcNAc moiety from the highenergy donor uridine 5'-diphosphate (UDP)-GlcNAc to substrate proteins, whereas OGA hydrolyzes O-GlcNAc from proteins. Dysregulation of key proteins O-GlcNAcylation has been demonstrated in the etiology of numerous varieties of tumors, including bladder cancer. ${ }^{17}$ However, the effects of O-GlcNAcylation in bladder cancer and if it can regulate NUSAP1 protein remain blear.

Based on the above, one of the objectives of this study is to explore the function of NUSAP1 in bladder cancer progression; the other is to determine the effects of O-GlcNAcylation on NUSAP1 protein expression and stability, as well as to investigate the role of O-GlcNAcylation of NUSAP1 on the tumorigenesis of bladder cancer cells. Our study expects to further clarify the functions of O-GlcNAcylation and NUSAP1 in the occurrence and development of bladder cancer, hoping to provide new idea for the invention of antitumor drugs.

\section{Materials and Methods}

\section{Patients and Tissue Samples}

Twenty-five matched fresh bladder cancer tissues and their adjoining non-tumor tissues were all obtained from Shanghai Tenth People's Hospital, between January 2015 to January 2017. All histological samples were identified by three seasoned pathologists without knowing the clinical data. And the study involved human samples was approved by the Human Research Committee of Shanghai Tenth People's Hospital and had been proceeded in accordance with the Helsinki Declaration.

\section{Immunohistochemistry (IHC)}

Paraffin sections of tissues were sliced into sections of 4 $\mu \mathrm{m}$ thickness by a slicing machine and processed just as follows: dewaxing and hydrating the tissues; incubation with $3 \% \mathrm{H}_{2} \mathrm{O}_{2}$ for $10 \mathrm{~min}$ at room temperature; antigen repairing with Tris-EDTA; blocking with 5\% goat serum (diluted in PBS); incubation overnight at $4^{\circ} \mathrm{C}$ with NUSAP1 primary antibody (1:50 dilution; No. PA524479, Thermofisher, MA, USA), followed by incubation with the Rabbit secondary antibody and washed with PBS. Chromogen 3, 3'-diaminobenzidine tetrachloride (Serva, Heidelberg, Germany) was used as a substrate. Harri's hematoxylin solution was used to stain the nucleus.

\section{Cell Culture}

Bladder cancer cell lines including HT-1376, 5637, and T24, and normal uroepithelium cell line SV-HUC-1 were all purchased from ATCC (VA, USA). HT-1376 and were grown in Eagle's Minimum Essential Medium (No. 30-2003, ATCC, VA, USA). Five thousand six hundred and thirty-seven cells were cultured in RPMI-1640 medium (Biological Industries, Kibbutz BeitHaemek, Israel), T24 cells were cultured in McCoy's 5a Medium (No. 30-2007, ATCC, VA, USA). The base medium for SV-HUC-1 cell line is ATCC-formulated F-12K Medium (No. 30-2004). The culture mediums for all cell lines contained $10 \%$ fetal bovine serum (FBS; Gibco, CA, USA), $100 \mathrm{U} / \mathrm{mL}$ of penicillin, and $100 \mu \mathrm{g} / \mathrm{mL}$ of streptomycin. And cells were cultured at $37^{\circ} \mathrm{C}$ in a humidified incubator with air charge of $5 \% \mathrm{CO}_{2}$.

Cell treated with $25 \mathrm{mM}$ PuGNAc (Sigma, St Louis, MO, USA) and $4 \mathrm{mM} \mathrm{GlcNAc} \mathrm{(Sigma,} \mathrm{St} \mathrm{Louis,} \mathrm{MO,}$ USA) for $24 \mathrm{~h}$ to up-regulate O-GlcNAc level. Cells were treated with $0.1 \mathrm{mg} / \mathrm{mL}$ of Cycloheximide (Chx; Sigma, $\mathrm{St}$ Louis, MO, USA) for $1,2,4,8$, and $24 \mathrm{~h}$ to explore protein stability, all respectively.

\section{Cell Transfection}

The over-expressing plasmid of NUSAP1 (OE-NUSAP1) was synthesized by GenePharma (Suzhou, Jiangsu, China). The shRNAs (No.TR30023) targeting human OGT gene and their controls were purchased from 
Origene (CA, USA). Lipofectamine 2000 obtained from Life Technologies (MA, USA) was used to perform cell transfection according to the manufacturer's instruction. For in vivo tumor formation assay, puromycin (for shRNA-OGT; $7 \mu \mathrm{g} / \mathrm{mL}$ ) and G418 (for OE-NUSAP1; 4 $\mu \mathrm{g} / \mathrm{mL}$ ) were used to screen cells with stable transfection.

\section{Real-Time Polymerase Chain Reaction (RT-PCR)}

Total RNA was isolated from bladder cancer tissues and cell lines using Trizol Reagent (Invitrogen, Carlsbad, CA, USA) in accordance with the manufacturer's instructions. cDNA was generated using Reverse Transcription System (Promega, Thermofisher, MA, USA). RT-PCR was performed by using AccessQuick ${ }^{\mathrm{TM}}$ RT-PCR System (Promega, Thermofisher, MA, USA). Primer sequences used in this study were as follows: NUSAP1 (F): 5'-GTGTGGACCCTGACTCACAG -3', (R): 5'- CTTCTTGGTGCTCGTCTGGT-3'; OGT (F): 5'CGGCTGCGTGTAGGATATGT-3'，(R): 5'-GCCATCACC TTCACTCGGAA-3'; GAPDH (F): 5'-GAGAAGGCTGGG GCTCATTT-3'; (R): 5'-AGTGATGGCATGGACTGTGG-3'.

\section{Western Blot Analysis (WB)}

Proteins from cells and tissue samples were obtained by using RIPA Lysis Buffer (Beyotime Biotechnology, Shanghai, China), and the protein extraction process was proceeded in ice in accordance with the instructions. After quantified with bicinchoninic acid (BCA) Protein Assay Kit (Thermo Fisher Scientific, MA, USA) and denatured in $100^{\circ} \mathrm{C}$ for $10 \mathrm{~min}$, protein samples from each group were separated on a $10 \%$ SDS-PAGE gel and transferred onto a PVDF membrane (Millipore, CA, USA). Then, the membranes were blocked by $5 \%$ nonfat milk for $1 \mathrm{~h}$ at room temperature and were incubated with the primary antibodies at $4^{\circ} \mathrm{C}$ overnight. Next day, the membranes were washed and incubated with the corresponding HRPlinked secondary antibodies (No. \#7074, \#7076, Cell Signaling Technology, CA, USA) at 1:10,000 dilution for $1 \mathrm{~h}$ at room temperature. The primary antibodies used for this study are anti-NUSAP1 (No. PA5-24479, Thermofisher, MA, USA) and anti-GAPDH (No. MA515738-BTIN, Thermofisher, MA, USA). The expression levels of proteins were measured by ECL (Millipore, CA, USA) and the ImageJ software (National Institutes of Health) was used for quantification after background subtraction.

\section{Cell Counting Kit-8 (CCK-8) Assay}

Cell proliferation was assessed by CCK-8 kit (Solarbio Life Sciences, Beijing, China) as described in the manufacturer's protocol.

\section{Flow Cytometry with Annexin V/ Propidium lodide (PI) Staining}

Flow cytometry with Annexin V/PI staining was used for cell apoptosis detection. Briefly, bladder cancer HT-1376 and T24 cells were grown in a 12-well plate. Forty-eight hours after the transfection, the cells were harvested and stained with PI and Annexin V (FITC) (Apoptosis Detection Kit, BD Biosciences, CA, USA) followed by detection with BDFACS CantoTM II. The percentage of cell population undergoing early and late apoptosis was assessed by FlowJo7.6 software.

\section{In vivo Tumor Formation Assay}

Animal experiments were conducted in accordance with ethical standards (Guide to Ethical Review of Laboratory Animal Welfare -GB/T 35892-2018) and approved by Institutional Animal Care and Use Committee of Shanghai Tenth People's Hospital. HT-1376 and T24 cells with stable transfection of shRNA-OGT, OENUSAP1, sh-OGT+OE-NUSAP1, and control vectors were subcutaneously injected into the both flanks of 4-week BALB/c nude mice (Shanghai SLAC Laboratory Animal Co, Ltd. China), with 5 mice in each group. Twenty-six days after injection, the mice were euthanized and tumors were taken out to measure their weight.

\section{Data Analysis}

The data statistical analyses of this study were performed with SPSS 22.0 software. The data are expressed as means \pm SD. The two-tailed Student's $t$-test and one-way ANOVA test were used to assess the difference between two groups or more. A value of $P<0.05$ was considered statistically significant.

\section{Results}

\section{NUSAPI is Highly Expressed in Bladder Cancer Tissues and Cell Line}

We firstly searched for Oncomine database to find the expression of NUASP1 in bladder cancer, 48 cases of bladder tissue samples and 28 cases of superficial bladder cancer tissue samples were included. Results showed that NUSAP1 was highly expressed in bladder cancer tissues as compared 
with that bladder tissues (Figure 1A). To confirm this, we also carried out IHC, WB, and RT-PCR to measure both the protein and mRNA levels of NUSAP1 in 25 bladder cancer tissues and the 25 paired adjacent non-tumor tissues, and bladder cancer tissues showed higher expression of NUSAP1 than the para-carcinoma tissues (Figure 1B-D). Similarly, compared with the normal uroepithelium SVHUC-1 cells, the mRNA and protein levels of NUSAP1 in bladder cancer HT-1376, 5637, and T24 cells were significantly elevated (Figure $1 \mathrm{E}$ and F). These results confirm that
NUSAP1 is over-expressed in bladder cancer tissues and cells in both protein and mRNA levels.

\section{Higher Expression of NUSAPI is Closely Associated with Bladder Cancer Patients' Aggressiveness and Bad Outcome}

We also evaluated the association of NUSAP1 expression pattern with patients' clinicopathological characteristics and prognosis. Thirty-two cases of bladder cancer patients
A

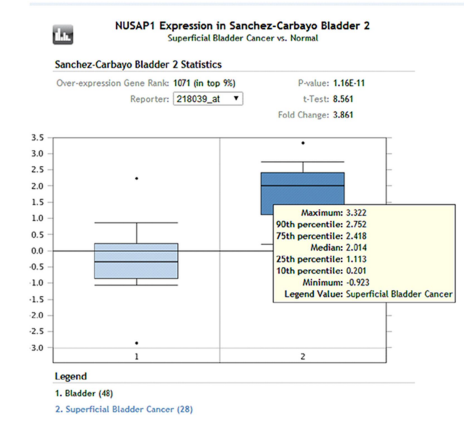

C

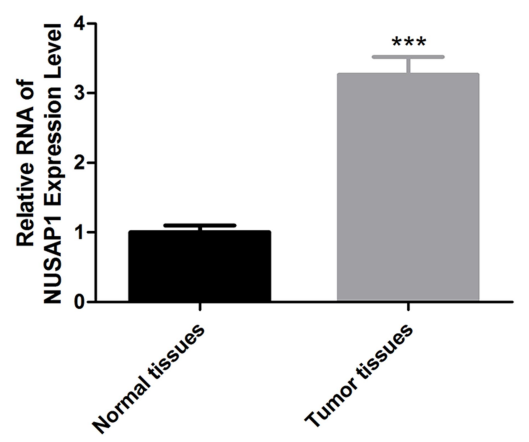

$\mathbf{F}$

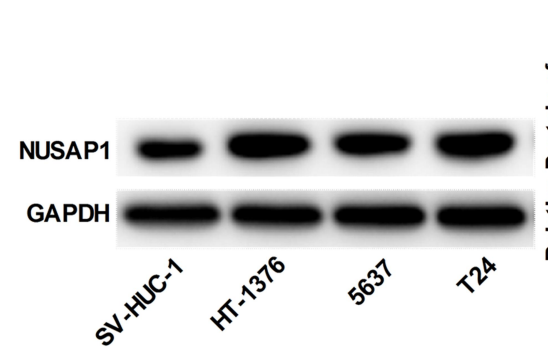

B
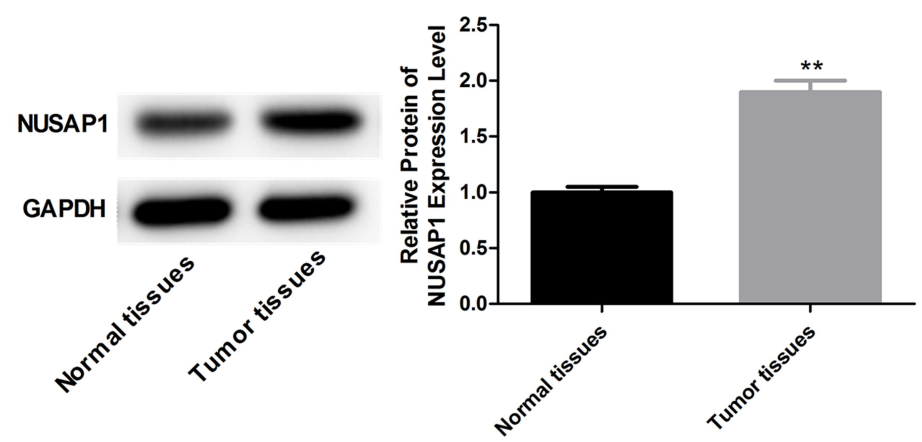

D

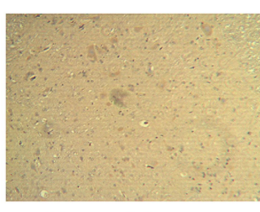

Normal tissues

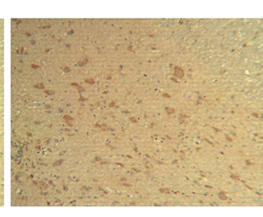

Tumor tissues

\section{E}

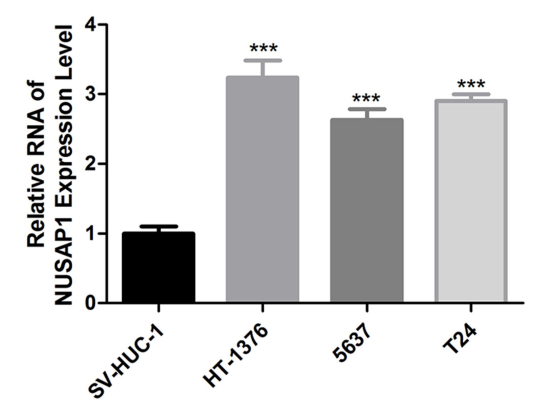

G

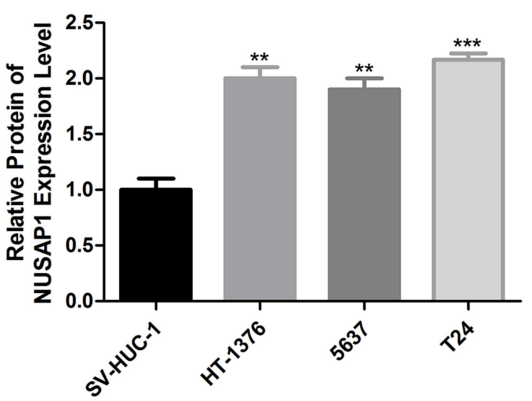

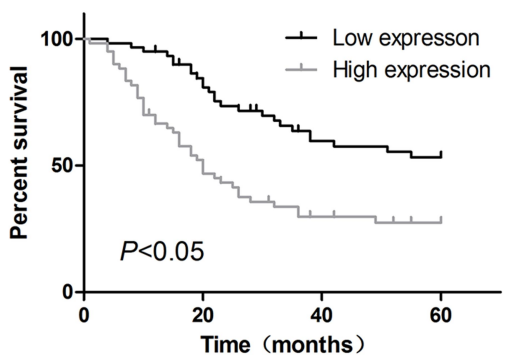

Figure I Overexpression of NUSAPI in bladder cancer tissues and cells. (A) Oncomine database showing the expression level of NUSAPI in bladder tissues ( $=48$ ) and superficial bladder cancer tissues $(n=28)(P<0.00 I)$. (B and $\mathbf{C})$ WB and RT-PCR was performed to determine the protein and mRNA levels of NUSAPI in 25 paired bladder cancer tissues and tissues adjacent to cancer. One representative figure was shown. ( $n=25, * * P<0.01$, $* * * P<0.00 I$, tumor tissues group vs normal tissues). (D) IHC was performed to detect NUSAPI expression using an-NUSAPI antibody. One representative figure was shown. (E and F) WB and RT-PCR was performed to determine the protein and mRNA levels of NUSAPI in normal uroepithelium cell line SV-HUC-I and bladder cancer cell line HT-I376, T24 and 5637. ( $\mathrm{n}=3$, $* * P<0.0 \mathrm{I}, * * * P<0.00 \mathrm{I}, \mathrm{HT}-\mathrm{I} 376$, T24 and 5637 group vs SV-HUC-I group). (G) Kaplan-Meier curve used to analysis the OS of bladder cancer patients with high ( $\mathrm{n}=28$ ) or low ( $\mathrm{n}=32$ ) expression of NUSAPI $(P<0.05)$. 
with NUSAP1 low expression and 28 cases with NUSAP1 high expression were chosen as the objects of our study. Statistical results indicate that patients with high NUSAP1 expression always showed larger tumors $(P=0.039)$, higher occurrence rate of lymphonodus metastasis $(P=0.003)$, more advanced TNM stage $(P=0.016)$, and lower differentiation $(P=0.009)$ (Table 1$)$. Moreover, the KaplanMeier curves were built to assess the difference of OS in patients with NUSAP1 high or low expression, and results showed that OS was obviously shorter in NUSAP1 high expression patients than in patients with NUSAP1 low expression $(P<0.05$, Figure $1 \mathrm{G})$.

\section{Overexpression of NUSAPI Promotes the Proliferation and Inhibits Apoptosis of Bladder Cancer Cells}

Next, we explored the effects of NUSAP1 in the progression of bladder cancer in vivo. OE-NUSAP1 significantly up-regulated the mRNA and protein levels of NUSAP1 in HT-1376 and T24 cells (Figure 2A and B). CCK-8 assay performed to detect cell proliferation showed that overexpression of NUSAP1 significantly enhanced cell

Table I Expression and Clinical Significance of NUSAPI in Bladder Cancer

\begin{tabular}{|c|l|l|l|l|}
\hline Groups & $\begin{array}{l}\text { Total } \\
\text { Cases }\end{array}$ & $\begin{array}{l}\text { Low } \\
\text { Expression } \\
\text { Cases }\end{array}$ & $\begin{array}{l}\text { High } \\
\text { Expression } \\
\text { Cases }\end{array}$ & P value \\
\hline $\begin{array}{c}\text { Gender } \\
\text { Male } \\
\text { Female }\end{array}$ & 49 & 26 & 23 & 0.929 \\
\hline $\begin{array}{c}\text { Age/years } \\
<60\end{array}$ & 6 & 5 & \\
$\geq 60$ & 43 & 25 & 18 & 0.235 \\
\hline $\begin{array}{c}\text { Size/cm } \\
\leq 3 \\
>3\end{array}$ & 17 & 7 & 10 & \\
\hline $\begin{array}{c}\text { Lymphonodus } \\
\text { metastasis } \\
\text { Yes } \\
\text { No }\end{array}$ & 22 & 16 & 6 & 0.022 \\
\hline $\begin{array}{c}\text { TNM } \\
\text { I-II } \\
\text { III-IV }\end{array}$ & 23 & 6 & 22 & 0.001 \\
\hline $\begin{array}{c}\text { Pathology } \\
\text { High } \\
\text { Medium } \\
\text { Low }\end{array}$ & 37 & 26 & 16 & 17 \\
\hline
\end{tabular}

proliferation in both HT-1376 and T24 cell lines, as compared with the control group (Figure $2 \mathrm{C}$ and $\mathrm{D}$ ). Besides, flow cytometry analysis with Annexin V/PI staining indicated that cell apoptosis (total early and late apoptosis cells) was repressed from $8.12 \%$ to $3.64 \%$ in HT-1376 cells, and from $9.40 \%$ to $5.32 \%$ in T24 cells when upregulation of NUSAP1 (Figure 2E). These results suggest that NUSAP1 functions as an oncogene in bladder cancer progression.

\section{O-GlcNAc Promotes NUSAPI Expression in Bladder Cancer}

Then, to investigate the relationship of O-GlcNAcylation and NUSAP1 in bladder cancer, we firstly determined O-GlcNAc expression in 3 matched bladder cancer tissues and para-carcinoma tissues. In accordance with the expression of NUSAP1, WB results showed that compared with the normal tissues group, the expression of O-GlcNAc was increased in the bladder cancer tissues (Figure 3A). Similarly, O-GlcNAc expression was significantly increased in bladder cancer HT-1376, 5637, and T24 cells when compared with SV-HUC-1 cells (Figure 3B). Then, GlcNAc and PuGNAc were added to the culture medium to increase O-GlcNAcylation in both BT-1376 and T24 cells, results showed that the protein levels of O-GlcNAc and NUSAP1 were all elevated when adding GlcNAc and PuGNAc to the cells, especially in GlcNAc + PuGNAc group (Figure 3C and D).

\section{O-GlcNAc Enhances the Stability of NUSAPI Protein in Bladder Cancer}

Subsequently, we assessed the stability of NUSAP1 protein after HT-1376 and T24 cells were treated with GlcNAc, PuGNAc, or GlcNAc + PuGNAc by using cycloheximide (Chx) to stop protein synthesis. And results showed that compared with the control group, treated cells with GlcNAc, PuGNAc or GlcNAc + PuGNAc obviously slowed down the degradation rate of NUSAP1 protein (Figure 4A). Moreover, we also measured the protein stability of NUSAP1 after O-GlcNAcylation was reduced by down-regulating OGT expression in HT-1376 and T24 cells. The expression of OGT was reduced about $50 \%$ when the cells were transfected shRNAs-OGT (Figure 4B), followed by reduced NUSAP1 stability (Figure 4C). Taken together, the above findings demonstrate that O-GlcNAc could slow down the degradation of NUSAP1 protein in bladder cancer cells. 
A

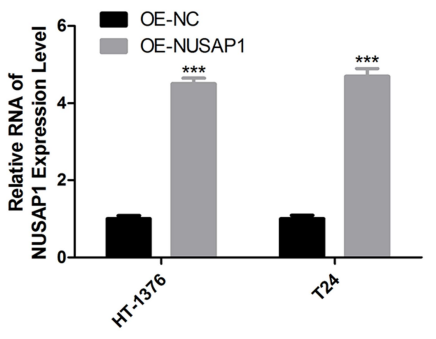

B

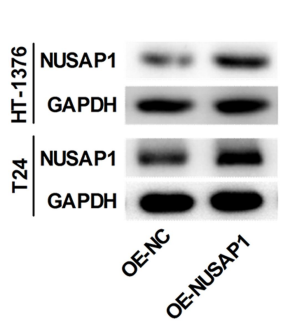

T24

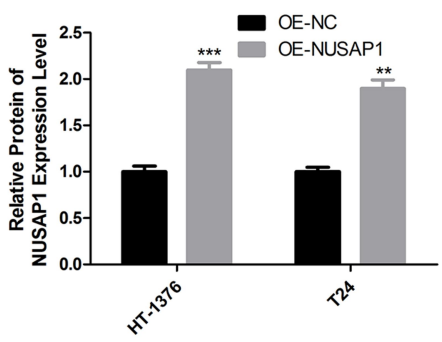

$\mathbf{E}$

C

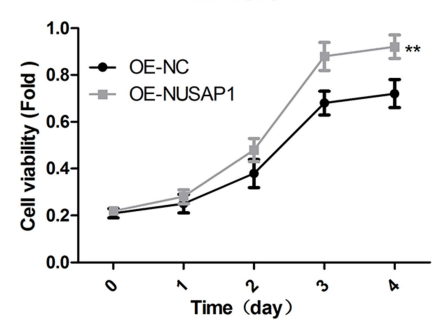

D

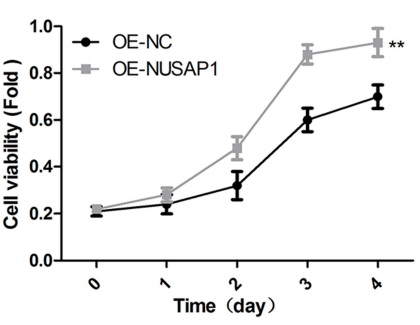

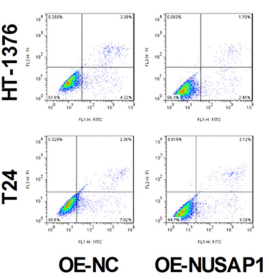

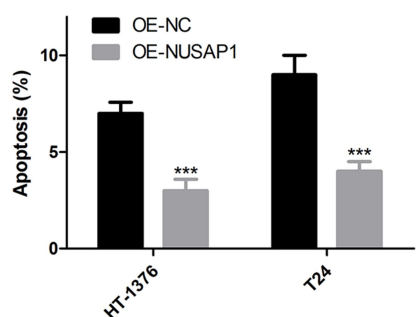

Figure 2 NUSAPI promoted the proliferation and repressed the apoptosis of bladder cancer cells. (A) HT-I376 and T24 cells were transfected with OE-NUSAPI and OENC, then A. $24 \mathrm{~h}$ after, RT-PCR was carried out to assess the mRNA level of NUSAPI ( $n=3, * * * P<0.001$, OE-NUSAPI group vs OE-NC group). (B) $48 \mathrm{~h}$ after, WB was used to evaluate the protein level of NUSAPI ( $n=3$, **P<0.0I, ${ }^{*} * *<0.00 \mathrm{I}$, OE-NUSAPI group vs OE-NC group). (C and $\left.\mathbf{D}\right)$ CCK-8 analysis of the proliferation of HT-I 376 and T24 cells after $0, I, 2,3$ and 4 days of the transfection, respectively $(n=3, * * P<0.0$ I, OE-NUSAPI group vs OE-NC group). (E) Flow cytometry with Annexin V/PI double staining was used to detect cell apoptosis $48 \mathrm{~h}$ after the transfection $(\mathrm{n}=3, * * * P<0.00 \mathrm{I}$, OE-NUSAPI group vs OE-NC group).

A
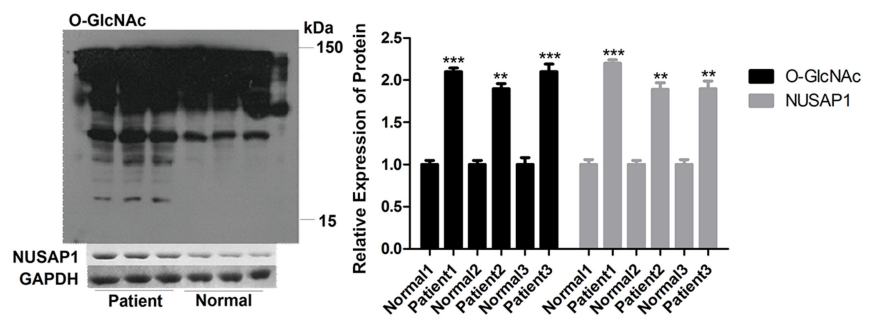

B
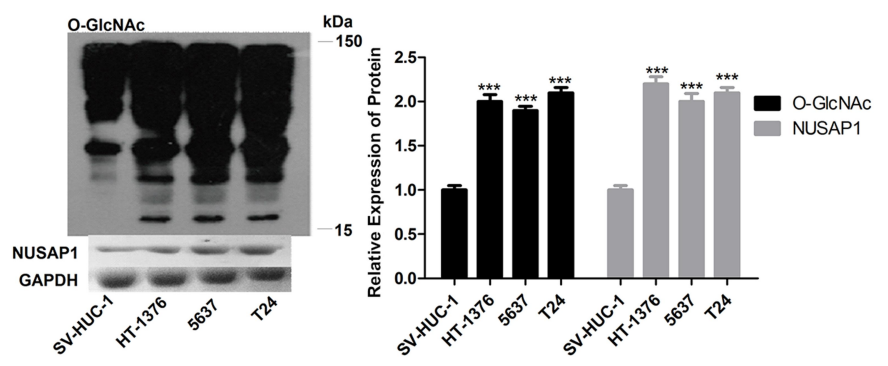

C
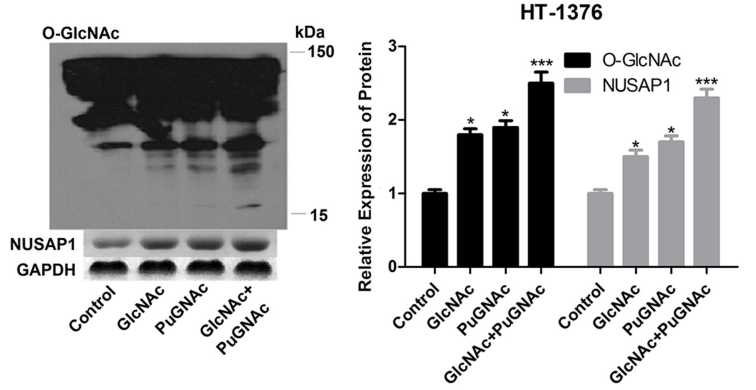

D

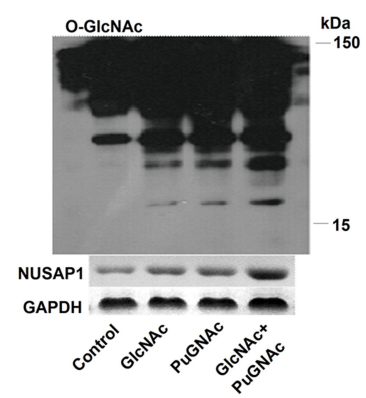

Figure 3 O-GlcNAc highly expressed in bladder cancers and increased NUSAPI expression. (A) WB analysis of the expression of NUSAPI and O-GlcNAc in 25 paired bladder cancer tissues and tissues adjacent to cancer. One representative figure was shown. $(n=25, * * P<0.01$, $* * * P<0.00 I$, tumor tissues group vs normal tissues). (B) WB was performed to determine the protein expression of NUSAPI and O-GIcNAc in normal uroepithelium cell line SV-HUC-I and bladder cancer cell line HT-I376, T24 and 5637. ( $\mathrm{n}=3, * * * P<0.00 \mathrm{I}, \mathrm{HT}-1376, \mathrm{~T} 24$ and 5637 group vs SV-HUC-I group). (C and D) HT-I 376 and T24 cells were treated with PuGNAc (25 mM), GlcNAc (4 mM) or PuGNAc + GlcNAc for $24 \mathrm{~h}$, then cells were collected for WB analysis to detect the expression of O-GlcNAc and NUSAPI ( $=3$, *P<0.05,***P<0.00I, PuGNAc, GlcNAc or PuGNAc + GlcNAc group vs control group). 
A

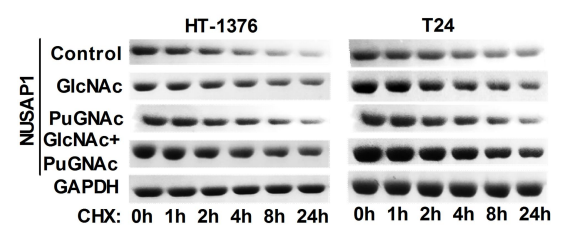

B

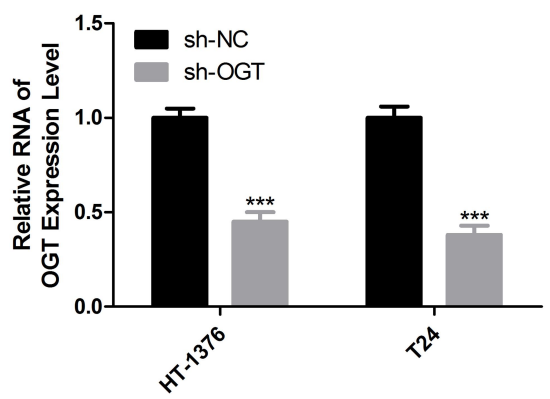

C

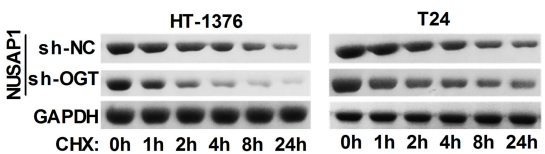

Figure 4 HT- 1376 and T24 cells were treated with GlcNAc $(4 \mathrm{mM})$, PuGNAc (25 mM) or GlcNAc + PuGNAc for $24 \mathrm{~h}$, then Chx ((I00 $\mu$ g/mL) was added to stop protein synthesis. (A) Then cells were lysed and equal amounts of protein were separated to WB to measure NUSAPI expression. (B) HT-I376 and T24 cells were transfected shOGT and sh-NC for $24 \mathrm{~h}$, then cells were collected and analysis for RT-PCR to determine the mRNA level of OGT ( $\mathrm{n}=3$, $* * * P<0.00 \mathrm{I}$, sh-OGT group vs sh-NC group). (C) WB analysis the expression of NUSAPI after OGT was knocked down $(n=3)$.

\section{Knockdown of OGT Inhibits the Progression of Bladder Cancer Through Down-Regulating NUSAPI Expression}

In the end, we investigated the effects of OGT in the development of bladder cancer and if NUSAP1 involved in this process. Knockdown of OGT decreased the expression of O-GlcNAc (Figure 5A). To the opposite of NUSAP1 up-regulation, down-regulation of OGT significantly repressed cell proliferation (Figure 5B and C) and promoted cell apoptosis (Figure 5D), whereas these roles were abolished when NUSAP1 was overexpressed in HT1376 and T24 cells (Figure 5B and D). In addition, tumorigenesis was significantly inhibited when HT-1376 and T24 cells were transfected shRNA-OGT when compared with the control group, which was reverse with NUSAP1 up-regulation, and tumorigenesis was significantly enhanced when T24 and HT-1376 cells were transfected sh-OGT and OE-NUSAP1 simultaneously (Figure 5E). These results indicated that down-regulation of OGT represses bladder cancer progression through downregulating NUSAP1 expression (Figure 6).

\section{Discussion}

Recently, increasing evidence has manifested that hyperO-GlcNAcylation is a general specialty for many kinds of cancers and promotes the transformation to more aggressive phenotypes, such as breast, ${ }^{18}$ ovarian, ${ }^{19}$ prostate, ${ }^{20}$ colorectal, ${ }^{21,22}$ chronic lymphocytic leukemia, ${ }^{23}$ and so on. ${ }^{24}$ In bladder cancer, Rozanski et $\mathrm{al}^{17}$ reported that OGT mRNA is detectable in $51.7 \%$ of 176 urine samples come from bladder cancer patients, but not discoverable in 143 healthy individuals. They also demonstrate that the mRNA level of OGT is closely correlated with the poorly differentiation (grade III, the most aggressive form) of cancer, ${ }^{17}$ suggesting that hyper-O-GlcNAcylation might involve in the occurrence and development of bladder cancer. However, the mechanism of O-GlcNAcylation remains largely unclear. In this study, we showed that O-GlcNAc level was elevated in bladder cancer cells and tissue samples, which could promote NUSAP1 expression and its protein stability and regulate the pathogenesis of bladder cancer in a NUSAP1 dependent manner.

NUSAP1 is identified as a protein related to cell cycle, and the mRNA and protein levels of which peak in G2 phase of mitosis, and drop rapidly after the split phase content in cells. ${ }^{25}$ Besides, NUSAPl expression is significantly elevated in the cells in proliferate stage; however, the high expression of NUSAPl can result in the stagnation of cells in $\mathrm{M}$ phase, and inhibition of its expression would lead to spindle body cannot be formed and chromosome cannot be separated. ${ }^{26,27}$ NUSAP1 is highly expressed in breast cancer, ${ }^{28}$ hepatocellular carcinoma, ${ }^{29}$ and pancreatic cancer, ${ }^{30}$ and functions as an oncogene in these cancers. To our best knowledge, the present study, for the first time, explored the expression of NUSAP1 and its effects on the occurrence and development of bladder cancer. We found that NUSAP1 was over-expression in bladder cancer tissues and cell lines, which high expression level was closely related to patients' aggressive clinical tumor phenotype and poor prognosis. Moreover, we observed that bladder cancer T24 and HT-1376 cells transfected with OE-NUSAP1 showed enhanced proliferation and decreased apoptosis when compared with the cells in the control group, suggesting that NUSAP1 promoted the progression of bladder cancer through inhibiting cell apoptosis and motivating cell growth. 
A

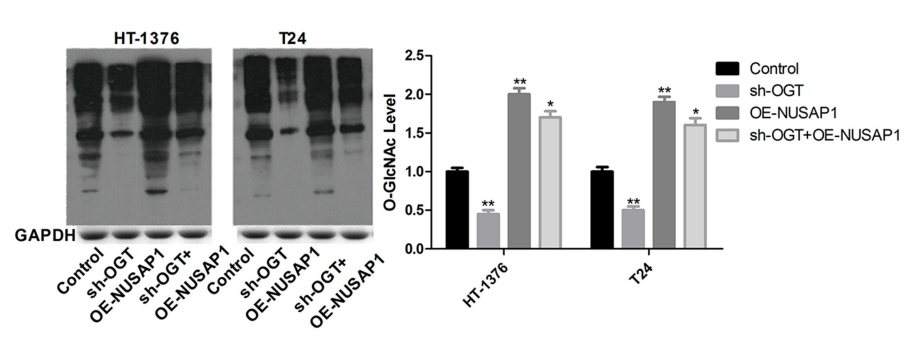

D

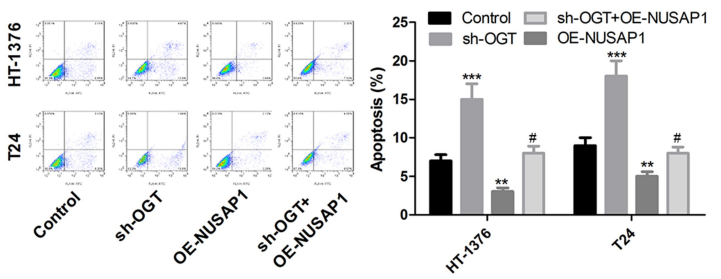

B

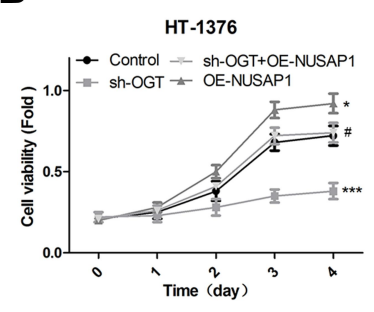

E

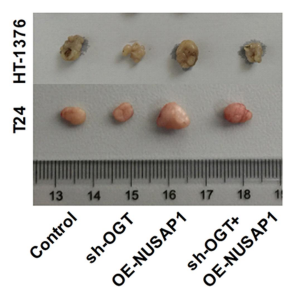

C

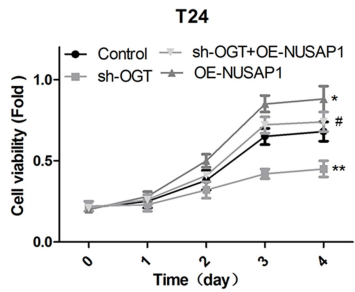

Figure 5 Down-regulation of OGT inhibited the proliferation and tumor formation and promoted the apoptosis of bladder cancer cells. HT-I376 and T24 cells were transfected control vector, OE-NUSAPI, sh-OGT and sh-OGT+OE-NUSAPI, then (A) WB analysis of the expression of O-GIcNAc after $48 \mathrm{~h}$ of the transfection. QPCR of the expression of O-GIcNAc $(n=3$, the experiment was repeated three times in each group; For HT-I $376 * *<<0.0$ I, sh-OGT group vs control group; $* * P<0.0$ I, OE-NUSAPI group vs control group; $* P<0.05$, sh-OGT+OE-NUSAPI group vs control group; For T24 $* * P<0.01$, sh-OGT group vs control group; $* * P<0.01$, OE-NUSAPI group vs control group; $* P<0.05$, sh-OGT+OE-NUSAPI group vs control group). (B and C) CCK-8 analysis of the proliferation of HT-I 376 and T24 cells after 0, I, 2,3 and 4 days of the transfection, respectively $(n=3$, the experiment was repeated three times in each group. $* * * P<0.00$ I, sh-OGT group vs control group; $* P<0.05$, OE-NUSAPI group vs control group; ${ }^{\# P>0.05}$, sh-OGT+OE-NUSAPI group vs sh-OGT group). (D) Flow cytometry with Annexin V/PI double staining was used to detect cell apoptosis $48 \mathrm{~h}$ after the transfection $(n=3$, the experiment was repeated three times in each group, For HT-I376 ***P<0.00I, sh-OGT group vs control group; $* * P<0.0$ I OE-NUSAPI group vs control group; ${ }^{*} P>0.05$, sh-OGT+OE-NUSAPI group vs control group; For T24 $* * * P<0.00$ I, sh-OGT group vs control group; $* * P<0.0$ I OE-NUSAPI group vs control group; ${ }^{\#} P>0.05$, sh-OGT+OE-NUSAPI group vs control group). (E) In vivo tumor formation assay was used to evaluate cell tumorigenesis ( $\mathrm{n}=3$, For $\mathrm{HT}-\mathrm{I} 376 * * \mathrm{P}<0.0 \mathrm{I}$, sh-OGT group vs control group; ${ }^{* * * P}<0.00 \mathrm{I}$, OE-NUSAPI group vs control group; ${ }^{*} P>0.05$, sh-OGT+OE-NUSAPI group vs control group; For T24 ${ }^{* *} P<0.0 \mathrm{I}$, sh-OGT group vs control group; $* * P<0.01$, OE-NUSAPI group vs control group; ${ }^{*} P>0.05$, sh-OGT+OE-NUSAPI group vs control group).

We also observed that the expression level of O-GlcNAc was elevated in bladder cancer cells and tissue samples, just as NUSAP1, indicating that O-GlcNAcylation might play an important role in bladder cancer. A unique trait of energy metabolism in cancer cells is a change from oxidative phosphorylation to the less efficient glycolytic pathway (Warburg effect), leading to elevated glucose and glutamine uptake, indicating that the increased glucose and glutamine uptake by cancer cells might be related to increased O-GlcNAc levels. ${ }^{24}$ Previous study has summarized the effects of O-GlcNAcylation on the expression and stability of proteins related to the modulation of cell proliferation, sustained angiogenesis, deregulated cellular energetics, invasion and metastasis, oxidative stress and metabolic stress and found that hyper-O-GlcNAcylation stimulation could increase the expression, stability, or activity of c-Myc, Cyclin D1, FoxM1, Snail, matrix Metalloproteinases (MMPs), OCT4, FoxO4, but reduced the expression, stability, or activity of E-cadherin, p27, phosphofructokinase1 (PFK1). ${ }^{24}$ Similarly, we showed that stimulation of O-GlcNAcylaltions with PuGNAc (an inhibitor of OGA), and GlcNAc (the substrate of O-GlcNAcylaltion) up-regulated NUSAP1 expression and its protein stability in both HT-1376 and T24 cells.

Furthermore, we explored the effects of O-GlcNAcylation in the progression of bladder cancer and determined if NUSAP1 took part in this process mediated by O-GlcNAcylation. shRNA targeting to human OGT gene was used to knock down OGT expression in bladder cancer HT-1376 and T24 cells. Results showed that down-regulation of OGT significantly inhibited the growth and tumorigenesis and promoted the apoptosis of HT-1376 and T24 cells, but these effects were abolished when NUSAP1 was overexpressed, indicating that O-GlcNacylation inhibition suppressed bladder cancer progression through down-regulation of NUSAP1.

\section{Conclusion}

In summary, this study demonstrates that hyperO-GlcNacylation and high NUSAP1 expression are observed in bladder cancer. O-GlcNacylation promotes NUSAP1 expression and stability, and inhibition of O-GlcNAcylation suppresses bladder cancer progression 


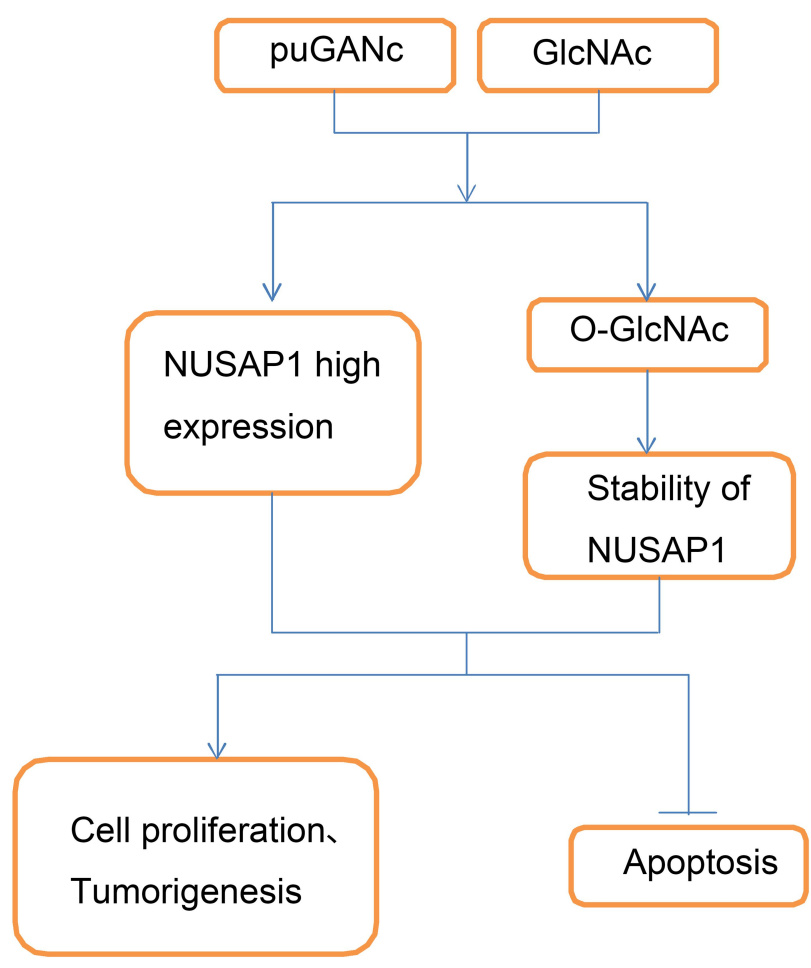

Figure 6 Schematic diagram of adverse effect of hyper-O-GlcNAcylation in the progression of bladder cancer. Up-regulation of O-GlcNAcylation level with PuGNAc and GlcNAc treatment promotes the expression and stability of NUSAPI protein, and accelerates the proliferation and tumorigenesis and represses the apoptosis of bladder cancer cells.

through down-regulation of NUSAP1. Our study proposes a comprehensive theory for the role of O-GlcNAcylation and NUSAP1 in bladder cancer.

\section{Abbreviations}

NUSAP1, nucleolar and spindle-associated protein 1; OGT, O-GlcNAc transferase; OGA, O-GlcNAcase.

\section{Data Sharing Statement}

The datasets generated in this study are available from WTZ on reasonable request.

\section{Ethics Approval and Consent to Participate}

The study was approved by the Ethics Committee at the Tenth People's Hospital of Shanghai. All patients who used the tissue in this study have signed informed consent forms.

\section{Author Contributions}

All authors contributed to data analysis, drafting or revising the article, have agreed on the journal to which the article will be submitted, gave final approval of the version to be published, and agree to be accountable for all aspects of the work.

\section{Disclosure}

The authors declare that they have no conflicts of interest for this work.

\section{References}

1. Siegel RL, Miller KD, Jemal A. Cancer statistics, 2016. CA Cancer J Clin. 2016;66(1):7-30. doi:10.3322/caac.21332

2. Torre LA, Bray F, Siegel RL, et al. Global cancer statistics, 2012. CA Cancer J Clin. 2015;65(2):87-108. doi:10.3322/caac.21262

3. Wu X, Xu B, Yang C, et al. Nucleolar and spindle associated protein 1 promotes the aggressiveness of astrocytoma by activating the hedgehog signaling pathway. J Exp Clin Cancer Res. 2017;36 (1):127. doi:10.1186/s13046-017-0597-y

4. Fang L, Zhang M, Chen L, et al. Downregulation of nucleolar and spindle-associated protein 1 expression suppresses cell migration, proliferation and invasion in renal cell carcinoma. Oncol Rep. 2016;36(3):1506-1516. doi:10.3892/or.2016.4955

5. Okamoto A, Higo M, Shiiba M, et al. Down-regulation of nucleolar and spindle-associated protein 1 (NUSAP1) expression suppresses tumor and cell proliferation and enhances anti-tumor effect of paclitaxel in oral squamous cell carcinoma. PLoS One. 2015;10(11): e0142252. doi:10.1371/journal.pone.0142252

6. Gordon CA, Gong X, Ganesh D, Brooks JD. NUSAP1 promotes invasion and metastasis of prostate cancer. Oncotarget. 2017;8 (18):29935-29950. doi:10.18632/oncotarget.15604

7. Gordon CA, Gulzar ZG, Brooks JD. NUSAP1 expression is upregulated by loss of RB1 in prostate cancer cells. The Prostate. 2015;75 (5):517-526. doi:10.1002/pros.22938

8. Chen L, Yang L, Qiao F, et al. High levels of nucleolar spindle-associated protein and reduced levels of BRCA1 expression predict poor prognosis in triple-negative breast cancer. PLoS One. 2015;10(10):e0140572. doi:10.1371/journal.pone.0140572

9. Gulzar ZG, McKenney JK, Brooks JD. Increased expression of NuSAP in recurrent prostate cancer is mediated by E2F1. Oncogene. 2013;32(1):70-77. doi:10.1038/onc.2012.27

10. Apweiler R, Hermjakob H, Sharon N. On the frequency of protein glycosylation, as deduced from analysis of the SWISS-PROT database. Biochim Biophys Acta. 1999;1473(1):4-8. doi:10.1016/ S0304-4165(99)00165-8

11. Li Y, Wang L, Liu J, et al. O-GlcNAcylation modulates Bmi-1 protein stability and potential oncogenic function in prostate cancer. Oncogene. 2017;36(45):6293-6305. doi:10.1038/onc.2017.223

12. Srikanth B, Vaidya MM, Kalraiya RD. O-GlcNAcylation determines the solubility, filament organization, and stability of keratins 8 and 18. J Biol Chem. 2010;285(44):34062-34071. doi:10.1074/jbc. M109.098996

13. Torres CR, Hart GW. Topography and polypeptide distribution of terminal $\mathrm{N}$-acetylglucosamine residues on the surfaces of intact lymphocytes. Evidence for O-linked GlcNAc. J Biol Chem. 1984;259 (5):3308-3317.

14. Onodera Y, Nam JM, Bissell MJ. Increased sugar uptake promotes oncogenesis via EPAC/RAP1 and O-GlcNAc pathways. J Clin Invest. 2014;124(1):367-384. doi:10.1172/JCI63146

15. Butkinaree C, Park K, Hart GW. O-linked beta-N-acetylglucosamine (O-GlcNAc): extensive crosstalk with phosphorylation to regulate signaling and transcripti21qon in response to nutrients and stress. Biochim Biophys Acta. 2010;1800(2):96-106. doi:10.1016/j. bbagen.2009.07.018 
16. Zachara NE, Hart GW. O-GlcNAc a sensor of cellular state: the role of nucleocytoplasmic glycosylation in modulating cellular function in response to nutrition and stress. Biochim Biophys Acta. 2004;1673 (1-2):13-28. doi:10.1016/j.bbagen.2004.03.016

17. Rozanski W, Krzeslak A, Forma E, et al. Prediction of bladder cancer based on urinary content of MGEA5 and OGT mRNA level. Clin Lab. 2012;58(5-6):579-583.

18. Trinca GM, Goodman ML, Papachristou EK, et al. O-GlcNAcdependent regulation of progesterone receptor function in breast cancer. Horm Cancer. 2018;9(1):12-21. doi:10.1007/s12672-0170310-9

19. de Queiroz RM, Madan R, Chien J, Dias WB, Slawson C. Changes in O-linked N-acetylglucosamine (O-GlcNAc) homeostasis activate the p53 pathway in ovarian cancer cells. J Biol Chem. 2016;291 (36):18897-18914. doi:10.1074/jbc.M116.734533

20. Kamigaito T, Okaneya T, Kawakubo M, Shimojo H, Nishizawa O, Nakayama J. Overexpression of O-GlcNAc by prostate cancer cells is significantly associated with poor prognosis of patients. Prostate Cancer Prostatic Dis. 2014;17(1):18-22. doi:10.1038/pcan.2013.56

21. Guo H, Zhang B, Nairn AV, et al. O-linked N-acetylglucosamine (O-GlcNAc) expression levels epigenetically regulate colon cancer tumorigenesis by affecting the cancer stem cell compartment via modulating expression of transcriptional factor MYBL1. J Biol Chem. 2017;292(10):4123-4137. doi:10.1074/jbc.M116.763201

22. Phueaouan T, Chaiyawat P, Netsirisawan P, et al. Aberrant O-GlcNAc-modified proteins expressed in primary colorectal cancer. Oncol Rep. 2013;30(6):2929-2936. doi:10.3892/or.2013.2794

23. Shi Y, Tomic J, Wen F, et al. Aberrant O-GlcNAcylation characterizes chronic lymphocytic leukemia. Leukemia. 2010;24 (9):1588-1598. doi:10.1038/leu.2010.152
24. Ma Z, Vosseller K. O-GlcNAc in cancer biology. Amino Acids. 2013;45(4):719-733. doi:10.1007/s00726-013-1543-8

25. Li L, Zhou Y, Sun L, et al. NuSAP is degraded by APC/C-Cdh1 and its overexpression results in mitotic arrest dependent of its microtubules' affinity. Cell Signal. 2007;19(10):2046-2055. doi:10.1016/j. cellsig.2007.05.017

26. Raemaekers T, Ribbeck K, Beaudouin J, et al. NuSAP, a novel microtubule-associated protein involved in mitotic spindle organization. J Cell Biol. 2003;162(6):1017-1029. doi:10.1083/ jcb.200302129

27. Ribbeck K, Groen AC, Santarella R, et al. NuSAP, a mitotic RanGTP target that stabilizes and cross-links microtubules. Mol Biol Cell. 2006;17(6):2646. doi:10.1091/mbc.e05-12-1178

28. Chen DT, Nasir A, Culhane A, et al. Proliferative genes dominate malignancy-risk gene signature in histologically-normal breast tissue. Breast Cancer Res Treat. 2010;119(2):335-346. doi:10.1007/s10549009-0344-y

29. Satow R, Shitashige M, Kanai Y, et al. Combined functional genome survey of therapeutic targets for hepatocellular carcinoma. Clin Cancer Res. 2010;16(9):2518-2528. doi:10.1158/1078-0432.CCR$09-2214$

30. Kokkinakis DM, Liu X, Neuner RD. Modulation of cell cycle and gene expression in pancreatic tumor cell lines by methionine deprivation (methionine stress): implications to the therapy of pancreatic adenocarcinoma. Mol Cancer Ther. 2005;4(9):1338-1348. doi:10.1158/1535-7163.MCT-05-0141

\section{Publish your work in this journal}

OncoTargets and Therapy is an international, peer-reviewed, open access journal focusing on the pathological basis of all cancers, potential targets for therapy and treatment protocols employed to improve the management of cancer patients. The journal also focuses on the impact of management programs and new therapeutic agents and protocols on patient perspectives such as quality of life, adherence and satisfaction. The manuscript management system is completely online and includes a very quick and fair peer-review system, which is all easy to use. Visit http://www.dovepress.com/ testimonials.php to read real quotes from published authors 